\title{
Current trends in gene therapy for retinal diseases (Review)
}

\author{
ANDREEA DANA MORARU ${ }^{1,2}$, DĂNUȚ $\operatorname{COSTIN}^{1,2}$, RALUCA EUGENIA IORGA $^{1,2}$, MIHNEA MUNTEANU $^{3 *}$, \\ RADU LUCIAN MORARU ${ }^{4 *}$ and DANIEL CONSTANTIN BRANISTEANU ${ }^{1,5}$ \\ ${ }^{1}$ Department of Ophthalmology, 'Grigore T. Popa’ University of Medicine and Pharmacy, 700115 Iași; \\ ${ }^{2}$ Department of Ophthalmology, 'N. Oblu' Clinical Hospital, 700309 Iași; ${ }^{3}$ Department of Ophthalmology, \\ 'Victor Babeș' University of Medicine and Pharmacy, 300041 Timișoara; ${ }^{4}$ Department of Otorhinolaryngology, \\ 'Transmed Expert' Medical Center, 700011 Iași; ${ }^{5}$ Department of Ophthalmology, \\ 'Retina Center' Eye Clinic, 700126 Iași, Romania
}

Received August 11, 2021; Accepted September 10, 2021

DOI: $10.3892 / \mathrm{etm} .2021 .10948$

\begin{abstract}
The eye is considered an effective target for genetic therapy, as it has a privileged immune status, it is easily accessed for medication delivery and it is affected by a number of inherited disorders. In particular, the retina is considered for gene therapy due to the fact that it can be visualized with ease, it does not have lymphatic vessels, nor a direct blood network for the outer layers and its cells do not divide after birth, and thus transgene expression is not affected. As gene therapy is currently on a continuously progressive development trend, this emerging field of gene manipulation techniques has yielded promising results. This involves the development of treatments for a number of debilitating and blinding diseases, which were to date considered intractable. However, numerous unanswered questions remain as regards the long-term efficacy and safety profile of these treatments. The present review article discusses the current research status regarding genetic manipulation techniques aimed at addressing visual impairment related to retinal disorders, both inherited and degenerative.
\end{abstract}

\section{Contents}

1. Introduction

2. Vectors

3. Delivery routes

4. Gene therapy for inherited retinal diseases

5. Gene therapy for age-related macular degeneration

6. Conclusions and future perspectives in gene therapy

Correspondence to: Dr Raluca Eugenia Iorga, Department of Ophthalmology, 'Grigore T. Popa' University of Medicine and Pharmacy, 16 Universitatii Street, 700115 Iași, Romania

E-mail: oftalmoconsultiasi@yahoo.ro; ralucadanulescu@yahoo.com

*Contributed equally

Key words: gene therapy, inherited retinal diseases, age-related macular degeneration, viral vectors, gene manipulation techniques

\section{Introduction}

Gene therapy is currently on a continuously progressive trend and over the past two decades, promising advancements have been made in the treatment of inherited and previously intractable diseases. Gene therapy techniques have in common the insertion of a foreign DNA fragment into host cells, aiming to modify the expression of proteins by the target cells. In order to deliver a modified gene to the target cell, the following requirements are warranted: i) Beneficial effects for the host suffering from a genetic mutation; ii) a cloned copy of the gene that is linked to the particular condition; iii) a system capable of promoting the transcription of the gene into the targeted cells; and iv) a vector which will carry the DNA to the cell, usually a virus, the virulence characteristics of which have been removed (1).

The eye is an effective target for genetic therapy, as it has a privileged immune status, it is easily accessed for medication delivery and it is affected by a number of inherited disorders. The retina is in particular considered for gene therapy due to the fact that it can be visualized with ease, it does not have lymphatic vessels, nor a direct blood network for the outer layers and its cells do not divide after birth, and thus the transgene expression is not affected. Over the past two decades, major improvements in surgical techniques necessary for the delivery of modified genes to the retinal tissue have been achieved, thus contributing to the development of novel and revolutionary therapeutic strategies $(1,2)$.

Genetic manipulation techniques include the following: Gene inactivation, gene augmenting and gene editing. The aim of gene inactivation is to block the production of an abnormal protein that is produced in the target cell and replace it with a therapeutic protein. This technique is mostly used in inherited retinal diseases that are associated with gain-of-function. Gene augmentation is particularly used in diseases characterized by loss-of-function, thus aiming at replacing a 'missing' protein in the target cell. Gene editing consists of marking DNA in the target cell for replacement. The technique is known as clustered regularly interspaced short palindromic repeats (CRISPR) and uses RNA linked to the Cas9 enzyme, in order to identify, cut and remove specific portions of DNA 
that are to be replaced (1). This technique has the disadvantage of possibly affecting other portions of DNA, thus creating novel mutations.

In order to assess the state of knowledge regarding genetic therapy for retinal diseases, the present study used MEDLINE/PubMed as the main biomedical database for research, using key words, such as gene therapy, inherited retinal diseases, retinal dystrophies, viral vectors, gene manipulation techniques and age-related macular degeneration (AMD). From $>800$ articles, the authors selected a total of 35 studies for review, the majority of which were published between 2006 and 2020. The articles were selected based on their relevance for each subchapter taken into discussion in the present review, and also based on the elements of novelty brought by each study, as more recent ones were preferred for inclusion.

\section{Vectors}

The delivery of the genetic material inside the target cell can be achieved by viral or non-viral methods. As viruses naturally infect human cells and insert their genetic material into the host cell nucleus, they can be considered a very efficient vector for genetic manipulation. The pathogenic viral genes are removed and the virus is used to insert the therapeutic gene inside the target cell. Lentiviruses and retroviruses are able to integrate their genetic material directly into the host genome. Herpes viruses and adenoviruses insert their genetic material as extrachromosomal episomes (1).

Lentiviruses have a single strand of RNA and are able to integrate their genetic material inside the chromosomes of the host, thus being capable of replicating continuously following a single administration, even in dividing cells, and sustaining long-term and stable transgene expression, even for large genes up to $10 \mathrm{~kb}$. A possible disadvantage in using lentiviruses for gene delivery is insertional mutagenesis, which may lead to the alteration of different genes with either the compromising of the cell's viability or the continuous replication and formation of a tumor (1).

Adenoviruses are double-stranded DNA viruses, which as vectors are able to transport the largest amount of genetic material of any viral vectors, up to $37 \mathrm{~kb}$. These viruses can infect dividing and non-dividing cells as well; however, their genetic material remains in the episomes, and thus the risk of insertional mutagenesis is significantly lower. Adenoviruses 2 and 5 are the most frequently used serotypes, which are able to transduce cells of the retinal pigment epithelium (RPE) cells and in some cases, photoreceptors. However, the disadvantage is that due to the lack of integration of the genetic material into the target cell genome, the genetic information inside the episomes is diluted with each mitosis cycle of dividing cells. Another drawback is the important immune response stimulated by the adenoviruses inside the organism of the host, which is able to remove all host cells that express adenovirus proteins via cytotoxic T-cell intervention, thus also eliminating the genetic information required for transduction. In order to elude the host immune response, helper dependent adenoviruses have been developed (2).

In ocular gene therapy, adeno-associated viral vectors are currently widely used. These are small and non-enveloped DNA viruses that belong to the Parvoviridae family and are non-pathogenic as they require a helper virus for replication. Similar to adenoviruses, they can infect both dividing and non-dividing cells and their genetic material remains inside the episomes. The capacity adeno-associated viral vector for packaging genetic material is only $4.8 \mathrm{~kb}$ and certain large genes cannot be inserted inside their genome. Serotypes 1, 2, 4, 5, 6, 7, 8 and 9 have tropism for the retinal tissues and are able to transduce cells of the RPE; however, the most frequently used serotypes in subretinal delivery are 2, 5 and 8 . Recombinant vectors can be used, which are hybrid or pseudo-typed adeno-associated viruses (AAVs), meaning they have components from various serotypes. Due to this characteristic, they are able to elude the immune response and have a higher transduction efficiency and an increased cellular tropism. Pseudo-types 2/1, 2/4 and 2/6, which recombine components from the correspondent serotypes, are efficient in transducing cells of the RPE, while pseudo-types $2 / 5,2 / 7,2 / 8$ and $2 / 9$ are mostly efficient in the transduction of photoreceptor cells $(3,4)$.

Over the past decade, second-generation vectors were introduced to ocular gene therapy. These are viruses that have a modified capsid structure, in order to prevent its degradation, to increase tropism for specific cells or to remove the antibody binding sites. Another category of second-generation vectors have a directed evolution, based on the accumulation of mutations, similar to natural selection, from which are selected the mutations that are proven to increase the transduction efficacy or render the vector capable of crossing biological barriers.

In order to increase cellular specificity, promoters and enhancers may be used. Following the integration of the genetic information brought to the target cell by the viral vectors, promoters, such as cytomegalovirus, allow the transcription of the desired transgene only in some specific cells. Thus, it is possible to target only the cells of the RPE or photoreceptors, while non-target cells will not transcribe the genetic material inserted by the vector, in the absence of promoter recognition $(5,6)$.

Non-viral methods consist of chemical and physical techniques of introducing the DNA inside the nucleus. Although they are less immunogenic, they are also less efficient in targeting cells in vivo. However non-viral techniques are able to deliver large genes into the host genome, they may be repeated and a larger dose may be used. Lipid- or polymer-based carriers are used in non-viral genetic manipulation and as these systems are not self-replicating, the administration needs to be repeated.

Liposomes consist of amphiphilic molecules, such as cholesterol and phospholipids, which can merge with the cellular membrane that also has a double-layered phospholipidic structure. Solid lipid nanoparticles have a lipid core surrounded by a layer of surfactants in an aqueous dispersion and may vary in size between 50 and $1,000 \mathrm{~nm}$, which renders it possible for them to be used in a subretinal injection delivery method.

Bioerodible polymers, such as, poly(lactic-co-glycolic acid) (PLGA), polyesters [poly(lactic acid); PLA], hyaluronic acid and chitosan are under investigation as possible carriers for genetic therapy (7-10).

\section{Delivery routes}

The most commonly used delivery routes for the novel genetic information towards the retinal tissue, with the aid of viral 
vectors are intravitreal injection, surgical subretinal injection and suprachoroidal administration.

The intravitreal injections are one of the safest and most widely used methods for therapy delivery in ophthalmology, particularly in the treatment of retinal diseases. When used to deliver genetic information to the retinal ganglion cells with the aid of AAVs, the intravitreal injections are not very efficient due to the presence of the vitreous and the internal limiting membrane. Vitrectomy and chemical-induced vitreous detachment by microplasmin, have been proved to aid vector penetration in retinal tissue (11).

The need for vitrectomy transforms the procedure into a surgical intervention. For this reason, an intravitreal injection for gene delivery is used when targeting the inner retinal layers or the retinal ganglion cell layer, as in Leber hereditary optic neuropathy (12).

In order to deliver genetic information to the cells of the RPE or photoreceptors, a subretinal injection is preferred. This method became the most frequently used for genetic therapy in ophthalmology, particularly following the approval of Luxturna by the FDA (13). After a complete vitrectomy is performed, a $41 \mathrm{G}$ soft-tipped cannula is used to reach the subretinal space. The therapeutic substance may be injected directly or it may be injected after first creating a subretinal balanced salt solution (BSS) bleb, which aids in the hydrodissection of the subretinal space, but can dilute the drug. The procedure is completed by fluid-air exchange. Inside the bleb, there is a high concentration of vector that enters the available cells, thus inducing a high expression of the desired transgene. However, cells situated outside the bleb will express a low amount of the transgene. Another possible disadvantage of this method of delivery may be the damaging of the photoreceptors due to the separation between the RPE and the photoreceptor outer segments, particularly in the foveal area. A subretinal injection may be difficult in eyes with subretinal fibrosis, which may lead to the appearance of macular holes, due to the high injection pressure (14).

The suprachoroidal administration is a less invasive procedure that delivers the therapeutic substance to a virtual space between the sclera and the choroid. The procedure has been proven to be safe and effective when using a triamcinolone injection, with the aid of microneedles (15).

In gene therapy, suprachoroidal delivery has been used in preclinical studies. In a previous study, An AAV8 vector that expresses a vascular endothelial growth factor (VEGF)-neutralizing protein, termed RGX-314, was proven efficient in suppressing the vascular leakage associated with vascular disease in a rat model. The transgene expression level was similar to that obtained with the subretinal injection. However, the various AAVs exhibited differential efficacy in inducing transgene expression, which may be an effect of anti-AAV antibodies present in the host organism (16).

\section{Gene therapy for inherited retinal diseases}

Gene therapy for inherited retinal diseases aims to replace a defective gene that is causing the illness with a normal one, delivered either in vivo or ex vivo, through cultured cells. The eye is an effective target for in vivo delivery, aided by viral vectors, as it is an immuno-privileged organ.
Current research on gene therapy for inherited retinal diseases is based on the gene augmentation technique. Following insertion, the normal gene is present in the episomes, inside the extrachromosomal DNA and the original, defective gene is still present. This approach is effective for inherited diseases that are associated with loss-of-function mutations (17).

The first gene therapy for an inherited retinal disease, approved in 2017, was voretigene neparvovec-rzyl (Luxturna, Spark Therapeutics) for retinal dystrophies with RPE65 mutations (13). If both RPE65 alleles present pathogenic mutations, patients develop autosomal recessive Leber congenital amaurosis or retinitis pigmentosa, presenting with progressive and severe visual loss, commencing from childhood.

The RPE65 gene is expressed in the RPE and it encodes for RPE-specific $65 \mathrm{kDa}$ protein, an enzyme necessary for converting 11-trans-retinil, which is not photoactive, to 11-cis-retinal, used by the photoreceptors during the visual cycle for visual pigment synthesis and regeneration $(18,19)$.

Luxturna is injected into the subretinal space and it involves the use of an AAV2 for the delivery of a human RPE65 to the cells of the RPE. This therapy has been shown to lead to an improvement in night vision, visual field and light sensitivity (20).

The clinical manifestations of pigmentary retinopathy are linked to the retinitis pigmentosa GTPase regulator (RPGR) gene mutation, which is involved in the regulation of ciliogenesis in photoreceptor cells (21). The loss-of-function mutation of the RPGR gene leads to the degeneration of the photoreceptors and the occurrence of either pigmentary retinopathy, cone dystrophy or cone-rod dystrophy. There are three early-phase clinical studies that are evaluating the efficacy of gene therapy in these photoreceptor dystrophies (ClinicalTrials.gov Identifier: NCT03116113, NCT03252847 and NCT03316560). The vectors used in these trials are AAV2 or AAV2/5 associated with rhodopsin kinase promoter, or AAV8 associated to codon optimized RPGR (22).

Another inherited retinal disease targeted for genetic therapy is X-linked retinoschisis. In this case, the trigger of the pathogenic mechanism is the loss-of-function of the retinoschisis 1 (RS1) gene, expressed by the photoreceptors and bipolar cells, which plays an important role in the maintenance of retinal structural integrity. Visual loss begins in childhood and it is caused by macular schisis, retinal detachment and vitreous hemorrhage (23). Two studies have been published using AAV and different gene variants, which are injected into the vitreous cavity $(24,25)$.

An AAV2 vector linked to a cone opsin promoter, which is administered via subretinal injection was used in a recent trial in order to induce the expression of the cyclic nucleotide-gated cation channel alpha-3 (CNG)A3 or CNGB3 gene in achromatopsia. The loss-of-function mutations of these genes generate the clinical manifestations of complete or incomplete achromatopsia. In complete achromatopsia, patients present with nystagmus, photophobia, a complete lack of color discrimination and loss of central vision from birth. These symptoms are less severe in incomplete achromatopsia (26-28).

In choroideremia, which is an X-linked inherited retinal disease, the defective CHM gene encoding for Rab escort protein 1 (REP-1) protein determines the loss-of-function of this protein, resulting in progressive RPE, choroidal and 
retinal atrophy. The loss of peripheral vision and nyctalopia begin in early childhood. A phase 2 (NCT02341807) and a phase 3 study (NCT03496012) are undergoing, both using AAV2 in order to deliver the CHM gene via subretinal injections, with both showing a good safety profile. In addition, a phase 1 study by 4D Molecular Therapeutics (NCT04483440) is being conducted and is studying the efficacy of an intravitreal injected AAV carrying a transgene (29).

Mutations of the ATP binding cassette subfamily A member 4 (ABCA4) gene stand at the base of the clinical features of Stargardt disease. This macular dystrophy causes progressive central visual loss, difficulties in dark adaptation and color vision loss. The ABCA4 gene, which is a large gene that cannot be contained in AAV vectors, encodes for both a membrane transporter implicated in phototransduction and for the removal of metabolites that result from this process. A trial sponsored by Sanofi uses an equine anemia lentivirus, StarGen, incapable of causing disease in humans, in order to transport the genetic material to the host cells. The results revealed that StarGen was well-tolerated following a subretinal injection (30).

Usher syndrome associates pigmentary retinopathy, congenital hearing loss and vestibular dysfunctions, caused by mutations in the myosin VIIA (MYO7A) gene. The MYO7A gene is also large and cannot be transported by AAV vectors, which is why the same lentivirus as for the Stargardt disease trial is used, via subretinal injection (31).

\section{Gene therapy for age-related macular degeneration}

AMD is a maculopathy resulting in the progressive degeneration of the retinal cells and central visual loss due to either atrophy in the non-exudative form or development of choroidal neovascular membranes in exudative AMD. Current treatments do not offer much hope for visual recovery in the atrophic AMD. Therapy for neovascular AMD consists of monthly administered anti-VEGF agents in intravitreal injections, being both costly and posing a burden for the patient and the health care system.

Gene therapy may represent an alternative to anti-VEGF therapy. Ongoing trials are assessing the possibility to treat AMD, which is one of the most common diseases responsible for visual loss among the elderly population worldwide, by using gene manipulation. The aim is to introduce a gene encoding for an anti-VEGF protein inside the host cells. Thus, RGX 314 and ADVM 022 are currently under study (NCT03066258 and NCT03748784).

A phase 1 trial was completed by Sanofi/Genzyme, using as vector an AAV2 which expresses a recombinant VEGF trap, FLT01 (NCT01024998). The binding domains for both VEGF and phosphatidylinositol glycan anchor biosynthesis class F protein (PIGF), the placental growth factor of the human VEGF receptor 1, are contained by the Fms related receptor tyrosine kinase 1 (FLT01) gene. A total of 19 patients were treated by intravitreal injections and to date, both the expression of the FLT01 gene and the morphological and functional outcomes have been inconsistent (32).

RGX 314, developed by REGENXBIO uses AAV8 for transporting a gene which encodes for a monoclonal antibody fragment able to neutralize VEGF. The gene is delivered specifically to retinal cells. The sustained production of an
anti-VEGF protein by retinal cells would be able to reduce the number of anti-VEGF agent intravitreal injections (33).

Another study, developed by Hemera Biosciences, is designed to assess the efficiency of HMR 59 (AAVCAGsCD59). An AAV2 vector is used, administered via intravitreal injection and the end point involves the inhibition of the complement cascade, by blocking the membrane attack complex (MAC). This therapy was able to reduce the proliferation of choroidal neovessels when injected into the eyes of mice in $60 \%$ of the cases and it is currently tested in treatment-naïve eyes diagnosed with AMD. MAC is involved in the formation of both choroidal neovascular membranes and geographic atrophy; thus, Hemera Biosciences is sponsoring two phase 1 studies, one for exudative AMD (NCT03585556) and one for non-exudative AMD (NCT03144999) (34).

Adverum Biotechnologies has an undergoing study on ADVM 022 genetic therapy for neovascular age related macular degeneration. ADVM 022 utilizes AAV7m8 as a vector for genetic material encoding for aflibercept protein and can be administered via intravitreal injection. A phase 1 study OPTIC enrolled patients that previously underwent anti-VEGF treatment, for ADVM 022 injection. At 34 weeks after receiving the treatment, ADVM 022 proved to be safe and effective, as none of the patients included needed additional anti VEGF intravitreal injections (35).

\section{Conclusions and future perspectives in gene therapy}

The majority of the current gene therapy trials asses the efficacy of gene augmentation in order to obtain a functional protein as expressed by the defective gene. However, in disorders that are caused by gain-of-function genetic errors, it is necessary to identify a strategy for gene inhibition. The CRISPR/CAS9 gene editing technique may be effective in achieving this goal. CAS9 nuclease and guide ARN can be delivered by viral vectors.

A number of eye diseases, including AMD or retinopathy of prematurity, are caused by polygenetic mutations. In order to develop an effective gene therapy for these types of diseases, various approaches are required. One of these may involve the targeting of neurotrophic factors, either by the expression of an anti-VEGF protein or via the inhibition of the degenerative pathway.

The AAVs are currently the most commonly used vectors in ophthalmic gene therapy, with AAV2 and AAV8, being the usual choice, due to their low immunogenicity and the reduced rate of side-effects as compared with other possible vectors. However, their capacity of genetic data transport is only $5 \mathrm{~kb}$ DNA (36).

Numerous important genes that encode functional and structural proteins are larger; thus, a different vector system is required. There are studies on animal models of Usher syndrome and Stargardt disease, regarding a double-vector system, each carrying a fragment of the encoded protein, which than suffer an intermolecular recombination in order to obtain the final product (37).

Another study direction in the gene therapy of retinal diseases is the assessment and the enhancement of the clinical response and the measurement of the transgene expression efficiency in the target tissue (38). The evaluation of visual function 
is mostly achieved by measuring the visual acuity and the visual field; however, these investigations do not completely reflect the function of the entire retina. Electrophysiology testing and optical coherence tomography could improve the morphological and functional results assessment. A specific multi-luminance mobility test was developed for the Leber congenital amaurosis patients that receive gene therapy in order to evaluate their night blindness (39).

In the field of gene therapy for retinal diseases, a number of unanswered questions remain. These are regarding the safety profile of the promoters, and the unanticipated side-effects of gene manipulation that may develop in the long-term, as well as the economical implication of this type of complex therapy. Nonetheless, the emerging field of gene therapy has yielded promising results in finding cures for a number of debilitating and blinding diseases, which were considered intractable to date.

\section{Acknowledgements}

Not applicable.

\section{Funding}

No funding was received.

\section{Availability of data and materials}

Not applicable.

\section{Authors' contributions}

All authors (ADM, DC, REI, MM, RLM and DCB) contributed equally to the study design, and participated in the preparation of the manuscript and the review process. DC, RLM and REI contributed to the collection of the relevant literature, data analysis and critical interpretation. ADM, MM and DCB conceived the review and modified the manuscript. DC and DCB confirm the authenticity of all the raw data. All authors have read and approved the final version of the manuscript.

\section{Ethics approval and consent to participate}

Not applicable.

\section{Patient consent for publication}

Not applicable.

\section{Competing interests}

The authors declare that they have no competing interests.

\section{References}

1. Yáñez-Muñoz RJ, Balaggan KS, MacNeil A, Howe SJ, Schmidt M, Smith AJ, Buch P, MacLaren RE, Anderson PN, Barker SE, et al: Effective gene therapy with nonintegrating lentiviral vectors. Nat Med 12: 348-353, 2006.

2. Parks RJ, Chen L, Anton M, Sankar U, Rudnicki MA and Graham FL: A helper-dependent adenovirus vector system: Removal of helper virus by Cre-mediated excision of the viral packaging signal. Proc Natl Acad Sci USA 93: 13565-13570, 1996.
3. Manfredi A, Marrocco E, Puppo A, Cesi G, Sommella A, Della Corte M, Rossi S, Giunti M, Craft CM, Bacci ML, et al: Combined rod and cone transduction by adenoassociated virus 2/8. Hum Gene Ther 24: 982-992, 2013.

4. Schön C, Biel M and Michalakis S: Retinal gene delivery by adeno associated virus (AAV) vectors: Strategies and applications. Eur J Pharm Biopharm 95: 343-352, 2015.

5. Dalkara D, Byrne LC, Klimczak RR, Visel M, Yin L, Merigan WH, Flannery JG and Schaffer DV: In vivo-directed evolution of a new adenoassociated virus for therapeutic outer retinal gene delivery from the vitreous. Sci Transl Med 5: 189ra76, 2013.

6. Macé E, Caplette R, Marre O, Sengupta A, Chaffiol A, Barbe P Desrosiers M, Bamberg E, Sahel JA, Picaud S, et al: Targeting channelrhodopsin-2 to ON-bipolar cells with vitreally administered AAV restores $\mathrm{ON}$ and $\mathrm{OFF}$ visual responses in blind mice. Mol Ther 23: 7-16, 2015.

7. Kiss S: A novel approach to ocular gene therapy: Evaluation of suprachoroidally administered non-viral DNA nanoparticles. In: 42nd Annual Meeting of the Macula Society. The Macula Society, Bonita Spring, FL, 2019.

8. Ramamoorth $M$ and Narvekar A: Non viral vectors in gene therapy-an overview. J Clin Diagn Res 9: GE01-GE06, 2015.

9. Pitkanen L, Ruponen M, Nieminen J and Urtti A: Vitreous is a barrier in nonviral gene transfer by cationic lipids and polymers. Pharm Res 20: 576-583, 2003.

10. Bloquel C, Bourges JL, Touchard E, Berdugo M, BenEzra D and Behar-Cohen F: Nonviral ocular gene therapy: Potential ocular therapeutic avenues. Adv Drug Deliv Rev 58: 1224-1242, 2006.

11. Ivanova E, Hwang GS, Pan ZH and Troilo D: Evaluation of AAV-mediated expression of Chop2-GFP in the marmoset retina. Invest Ophthalmol Vis Sci 51: 5288-5296, 2010.

12. Tshilenge KT, Ameline B, Weber M, Mendes-Madeira A, Nedellec S, Biget M, Provost N, Libeau L, Blouin V, Deschamps JY, et al: Vitrectomy before intravitreal injection of AAV2/2 vector promotes efficient transduction of retinal ganglion cells in dogs and nonhuman primates. Hum Gene Ther Methods 27: 122-134, 2016.

13. Darrow JJ: Luxturna: FDA documents reveal the value of a costly gene therapy. Drug Discov Today 24: 949-954, 2019.

14. Davis JL, Gregori NZ, MacLaren RE and Lam BL: Surgical technique for subretinal gene therapy in humans with inherited retinal degeneration. Retina 39 (Suppl 1): S2-S8, 2019.

15. Campochiaro PA, Wykoff CC, Brown DM, Boyer DS, Barakat M, Taraborelli D and Noronha G; Tanzanite Study Group: Suprachoroidal triamcinolone acetonide for retinal vein occlusion: Results of the tanzanite study. Ophthalmol Retina 2: 320-328, 2018.

16. Ding K, Shen J, Hafiz Z, Hackett SF, Silva RLE, Khan M, Lorenc VE, Chen D, Chadha R, Zhang M, et al: AAV8-vectored suprachoroidal gene transfer produces widespread ocular transgene expression. J Clin Invest 129: 4901-4911, 2019.

17. DiCarlo JE, Mahajan VB and Tsang SH: Gene therapy and genome surgery in the retina. J Clin Invest 128: 2177-2188, 2018.

18. Tang PH, Buhusi MC, Ma JX and Crouch RK: RPE65 is present in human green/red cones and promotes photopigment regeneration in an in vitro cone cell model. J Neurosci 31: 18618-18626, 2011.

19. Miraldi Utz V, Coussa RG, Antaki F and Traboulsi EI: Gene therapy for RPE65-related retinal disease. Ophthalmic Genet 39: 671-677, 2018.

20. Gao J, Hussain RM and Weng CY: Voretigene neparvovec in retinal diseases: A review of the current clinical evidence. Clin Ophthalmol 14: 3855-3869, 2020.

21. Zemba M, Zaharia AC and Dumitrescu OM: Association of retinitis pigmentosa and advanced keratoconus in siblings. Rom J Ophthalmol 64: 313-321, 2020.

22. Martinez-Fernandez De La Camara C, Nanda A, Salvetti AP, Fischer MD and MacLaren RE: Gene therapy for the treatment of X-linked retinitis pigmentosa. Expert Opin Orphan Drugs 6: 167-177, 2018.

23. Potop V, Coviltir V, Schmitzer S, Corbu C, Ionescu IC, Burcel M and Dăscălescu D: The relationship between corneal hysteresis and retinal ganglion cells-a step forward in early glaucoma diagnosis. Med Sci Monit 26: e924672, 2020.

24. Cukras C, Wiley HE, Jeffrey BG, Sen HN, Turriff A, Zeng Y, Vijayasarathy C, Marangoni D, Ziccardi L, Kjellstrom S, et al: Retinal AAV8-RS1 gene therapy for X-linked retinoschisis: Initial findings from a phase I/IIa trial by intravitreal delivery. Mol Ther 26: 2282-2294, 2018. 
25. Mishra A, Vijayasarathy C, Cukras CA, Wiley HE, Sen HN Zeng Y, Wei LL and Sieving PA: Immune function in X-linked retinoschisis subjects in an AAV8-RS1 phase I/IIa gene therapy trial. Mol Ther 29: 2030-2040, 2021.

26. Michalakis S, Schön C, Becirovic E and Biel M: Gene therapy for achromatopsia. J Gene Med: 19, 2017 doi: 10.1002/jgm.2944

27. Hassall MM, Barnard AR and MacLaren RE: Gene therapy for color blindness. Yale J Biol Med 90: 543-551, 2017.

28. Nicoară SD, Şuşman S, Tudoran O, Bărbos O, Cherecheș G, Aștilean S, Potara M and Sorițău O: Novel strategies for the improvement of stem cells' transplantation in degenerative retinal diseases. Stem Cells Int 2016: 1236721, 2016.

29. Lam BL, Davis JL, Gregori NZ, MacLaren RE, Girach A, Verriotto JD, Rodriguez B, Rosa PR, Zhang X and Feuer WJ: Choroideremia gene therapy phase 2 clinical trial: 24 -month results. Am J Ophthalmol 197: 65-73, 2019.

30. Binley K, Widdowson P, Loader J, Kelleher M, Iqball S, Ferrige G, de Belin J, Carlucci M, Angell-Manning D, Hurst F, et al: Transduction of photoreceptors with equine infectious anemia virus lentiviral vectors: Safety and biodistribution of StarGen for Stargardt disease. Investig Ophthalmol Vis Sci 54: 4061-4071, 2013.

31. Zallocchi M, Binley K, Lad Y, Ellis S, Widdowson P, Iqball S, Scripps V, Kelleher M, Loader J, Miskin J, et al: EIAV-based retinal gene therapy in the shakerl mouse model for usher syndrome type 1B: Development of UshStat. PLoS One 9: e94272, 2014.

32. Heier JS, Kherani S, Desai S, Dugel P, Kaushal S, Cheng SH, Delacono C, Purvis A, Richards S, Le-Halpere A, et al: Intravitreous injection of AAV2-sFLT01 in patients with advanced neovascular age-related macular degeneration: A phase 1, open-label trial. Lancet 390: 50-61, 2017.
33. REGENXBIO Reports second quarter 2019 financial and operating results and additional positive interim phase I/ IIa trial update for RGX-314 for the treatment of wet AMD. REGENXBIO, Rockville, MD, 2019. http://ir.regenxbio.com/ news-releases/news-release-details/regenxbio-announces-additionalpositive-interim-phase-iiia-and/. Last Updated February 16, 2021.

34. Cashman SM, Ramo K and Kumar-Singh R: A non membrane-targeted human soluble CD59 attenuates choroidal neovascularization in a model of age related macular degeneration. PLoS One 6: e19078, 2011.

35. Grishanin R, Vuillemenot B, Sharma P, Keravala A, Greengard J, Gelfman C, Blumenkrantz M, Lawrence M, Hu W, Kiss S and Gasmi M: Preclinical evaluation of ADVM-022, a novel gene therapy approach to treating wet age-related macular degeneration. Mol Ther 27: 118-129, 2019.

36. Salganik M, Hirsch ML and Samulski RJ: Adeno-associated virus as a mammalian DNA vector. Microbiol Spectr 3: 10.1128/microbiolspec.MDNA3-0052-2014, 2015.

37. Trapani I, Puppo A and Auricchio A: Vector platforms for gene therapy of inherited retinopathies. Prog Retin Eye Res 43 108-128, 2014.

38. Lee JH, Wang JH, Chen J, Li F, Edwards TL, Hewitt AW and Liu GS: Gene therapy for visual loss: Opportunities and concerns. Prog Retin Eye Res 68: 31-53, 2019.

39. Chung DC, McCague S, Yu ZF, Thill S, DiStefano-Pappas J, Bennett J, Cross D, Marshall K, Wellman J and High KA: Novel mobility test to assess functional vision in patients with inherited retinal dystrophies. Clin Exp Ophthalmol 46: 247-259, 2018. International (CC BY-NC-ND 4.0) License. 